\title{
Creatividad, ocio y rendimiento académico en estudiantes de Ingeniería
}

\author{
Romina Cecilia Elisondo. Universidad Nacional de Río Cuarto. Consejo Nacional de \\ Investigaciones Científicas y Técnicas de Argentina \\ Analía Claudia Chiecher. Universidad Nacional de Río Cuarto. Consejo Nacional de \\ Investigaciones Científicas y Técnicas de Argentina \\ Paola Verónica Rita Paoloni. Universidad Nacional de Río Cuarto. Consejo Nacional de \\ Investigaciones Científicas y Técnicas de Argentina
}

Recepción: 24.01.2018 | Aceptado: 19.02.2018

Correspondencia a través de ORCID: Romina Cecilia Elisondo (iD ) 0000-0002-7841-9878

Citar: Elisondo, RC. Chiecher, AC. y Paoloni, PVR. (2018). Creatividad, ocio y rendimiento académico en estudiantes de Ingeniería. ReiDoCrea, 7, 28-42.

\section{Financiación: Consejo Nacional de Investigaciones Científicas y Técnicas (CONICET)}

Resumen: Investigaciones previas indican la importancia de la creatividad en el campo de la ingeniería. El objetivo del presente trabajo es analizar relaciones entre creatividad, ocio y rendimiento académico en estudiantes de Ingeniería de la Universidad Nacional de Río Cuarto (Argentina). El diseño del estudio fue no experimental descriptivo-correlacional y se realizó en dos momentos: febrero de 2015 y agosto de 2016. Participaron 132 estudiantes de la cohorte 2015 de las carreras de Ingeniería Mecánica, Química, Electricista y en Telecomunicaciones. Se utilizó el Test CREA, el Cuestionario de Acciones Creativas (CAC) y cuestionarios socio-demográficos y de ocio. Se observó que los estudiantes que participan en actividades de ocio serio obtienen puntajes superiores en creatividad. Las correlaciones entre rendimiento académico y creatividad fueron muy bajas. Sin embargo, los alumnos que rindieron más materias son los que tienen puntajes elevados en acciones creativas. Los resultados muestran que disponer de ciertas capacidades creativas parece ser un recurso importante en el oficio de ser estudiante de ingeniería. Los potenciales impactos de la investigación se orientan a desarrollar contextos educativos que promuevan diversidad de experiencias para contribuir en la construcción de trayectorias estudiantiles de logro.

Palabras clave: Creatividad | Ingeniería

Creativity, Leisure and Academic Performance in Engineering Students

Abstract: Previous research indicates the importance of creativity in the field of engineering. The objective of this article is to analyze relationships between creativity, leisure and academic performance in engineering students at the National University of Río Cuarto (Argentina). The design of the study was non-experimental descriptive correlational and was carried out at two different times: February 2015 and August 2016. The participants in the study were 132 students of mechanical, chemical, electrical and telecommunications engineering. The CREA Test, the Creative Actions Questionnaire (CAC) and sociodemographic and leisure questionnaires were used. It was observed that students who participate in serious leisure activities obtain higher scores in creativity. The correlations between academic performance and creativity were very low. However, the students who passed the most courses are those who have high scores in creative actions. The results show that having certain creative abilities seems to be an important resource for engineering students. The potential impacts of the study are aimed at developing educational contexts that promote diversity of experiences, contributing to the building of successful academic careers.

Keywords: Creativity | Engineering

\section{Introducción}

El escrito atiende a las relaciones entre creatividad, ocio y rendimiento académico en estudiantes de ingeniería, específicamente en jóvenes que ingresan en la universidad. Los estudiantes llegan a la universidad con experiencias construidas en contextos diversos, dentro y fuera de las instituciones educativas. Muchas de estas experiencias se construyen durante la participación en actividades de ocio estructuradas y sistemáticas. En investigaciones previas se ha observado que los estudiantes 
potencialmente más creativos participan de manera sistemática en actividades extracurriculares y de ocio (Elisondo y Donolo, 2015; Gómez y Lago, 2017; Nicolau, 2017).

El presente estudio se focaliza en alumnos ingresantes en Carreras de Ingeniería. Las ingenierías están actualmente en la mira, al menos en el contexto de Latinoamérica y particularmente en Argentina. El rápido crecimiento de la ciencia, la informática y la tecnología exigen una densidad de conocimiento técnico que no parece cubrirse con la cantidad de profesionales que egresan actualmente (Panaia, 2007; 2015). Haciéndose eco de tal escasez, las organizaciones gubernamentales han puesto en marcha políticas educativas y científicas para el incentivo de las Carreras de Ingeniería a través de diferentes programas y acciones, apuntando al objetivo de lograr mayor cantidad de ingenieros. En este contexto, atender a la formación de estudiantes de ingeniería así como a los contextos que potencialmente favorecerían mejores rendimientos parece una cuestión clave. La relevancia del estudio está dada no solo por los potenciales impactos educativos, sino también por las posibilidades de desarrollo humano y social que ofrecen la creatividad y el ocio (Csikszentmihalyi, 2001; Elizalde, Gomes, Bahia y Lacerda, 2015; Hegarty, 2009). Específicamente en estudios con alumnos universitarios se hallaron evidencias respecto de relaciones entre ocio y percepción de salud y bienestar (Cabeza, 2017; Pascucci, 2015).

El estudio se realizó en el contexto de una Facultad de Ingeniería de una Universidad pública, situada en el centro de Argentina. En dicha Facultad funciona desde el año 2005 el Laboratorio de Monitoreo de Inserción de Graduados (en adelante MIG). El MIG está integrado principalmente por investigadores del área de educación y depende de Secretaría Académica de la Facultad de Ingeniería. Su misión es la de recoger sistemáticamente, analizar y poner a disposición de la Secretaría Académica información sobre las trayectorias educativas, laborales y vitales de sus graduados, estudiantes y personas que abandonaron sus estudios para ejecutar políticas orientadas a promover la permanencia, el egreso y la inserción laboral. En la línea mencionada, en estudios previos se han relevado y analizado datos que permitieron caracterizar a la población de estudiantes de ingeniería, atendiendo a variables estructurales (sexo, edad, nivel educativo de los padres, ocupación de los padres, entre otras), trayectorias laborales paralelas al estudio y trayectorias académicas o de formación. Los principales resultados de dos censos estudiantiles realizados por el MIG en 2005 y 2014 con la población de estudiantes de ingeniería pueden consultarse en estudios realizados en el equipo de investigación (Moreno, Chieher y Paoloni, 2016; Paoloni, Chiecher y Sanchez, 2007).

También se han realizado estudios sobre los perfiles típicos de los estudiantes de ingeniería que logran trayectorias de éxito en el primer año universitario. En tal sentido, los datos sugieren que ser mujer, proceder de una familia donde se valora el estudio y donde los progenitores han completado el nivel secundario y/o universitario, estar motivado hacia el aprendizaje, atribuir los logros a causas internas, manejables y controlables, regular el aprendizaje, fijarse metas, planificar tiempos, recurrir y saber usar la ayuda de pares y docentes, contar con familias que operen como fuentes de apoyo y de sostén afectivo, emocional y también económico, encontrarse con docentes que proponen tareas desafiantes, de resolución de problemas reales, de aplicación de conocimientos, entre otros aspectos, parecen ser características comunes entre los estudiantes que consiguen seguir el ritmo estipulado por el plan de estudios de la carrera elegida (Chiecher, en prensa). Se ha atendido a características diversas de los estudiantes de ingeniería y al papel que potencialmente tienen en la configuración de trayectorias de logro o de abandono. Sin embargo, en el marco de investigaciones previas no se hizo foco en las variables que aquí se proponen tratar: las actividades de ocio en las que se involucran los jóvenes que llegan a estudiar 
ingeniería, las acciones creativas que pudieron haber desarrollado en distintos ámbitos previamente al ingreso en la universidad así como las potencialidades y capacidades creativas que los caracterizan.

La investigación se sustenta en perspectivas teóricas que destacan la importancia de estudiar la creatividad cotidiana, los desempeños de las personas en diferentes contextos de la vida diaria y en interrelación con los demás miembros de la comunidad (Long, 2014; Silvia et al., 2014). Específicamente en el estudio se atiende a grupos de alumnos de carreras de ingeniería, considerando que la creatividad es un requisito indispensable de este tipo de carreras. Alencar y Fleith (2008) analizaron factores que estimulan e inhiben la creatividad desde la perspectiva de estudiantes de ingeniería. Según los estudiantes, preparación, incentivo, inteligencia y autoconfianza son factores facilitadores de la creatividad. Respecto de los factores inhibidores, mencionan falta de flexibilidad, miedo de errores y falta de motivación. Las autoras sugieren promover contextos de enseñanza que no solo enfaticen el dominio de conocimientos específicos, sino también el fortalecimiento de la creatividad y las nuevas ideas. Según Cropley (2015), la ingeniería es fundamentalmente un proceso de resolución creativa de problemas. En este sentido, enfatiza la necesidad de promover la creatividad en la formación de ingenieros.

En estudios previos se ha observado que las personas que participan en actividades estructuradas de ocio obtienen puntajes superiores en las mediciones de creatividad respecto de quienes no participan (Elisondo y Donolo, 2016; Wolfradt y Pretz, 2001). Las relaciones entre creatividad y rendimiento académico son complejas y dependen de los grupos, los instrumentos de medición y los indicadores (Chamorro-Premuzic y Furnham, 2003; Xiaoxia Ai, 1999). Las investigaciones analizadas muestran resultados discordantes, en algunos casos se observan correlaciones moderadas positivas entre ambas variables (Limiñana et al., 2010; López- Calichs, 2006), en otros relaciones negativas (Kerstin, Martínez y Martín, 2015) y en algunos estudios, correlaciones muy bajas entre los constructos (Elisondo y Donolo, 2015; Balgiu y Adir, 2014; Fuentes Oseguera et. al, 2015; Gajda, 2016).

En el presente estudio se analizaron relaciones entre mediciones de creatividad, tanto de capacidades como de acciones creativas, y desempeños en actividades de ocio, considerando ocio casual y serio según la clasificación de Stebbins $(2011 ; 2012)$. Asimismo, se analiza la incidencia de variables socio-demográficas en las mediciones de creatividad, teniendo en cuenta que en estudios anteriores se han observado diferencias significativas según sexo y edad (Elisondo, 2015). Para el análisis de las actividades de tiempo libre se considera la perspectiva del ocio serio como contexto conceptual que sintetiza tres formas principales de ocio: serio, casual y basado en proyectos. El ocio serio refiere a la práctica sistemática de una actividad amateur, de voluntariado o un hobby, suficientemente sustancial, interesante y plena como para que el participante encuentre ahí un itinerario (de ocio), centrado en adquirir y expresar la combinación de habilidades, conocimientos y experiencia específicos de la actividad. El ocio casual es la actividad placentera inmediata e intrínsecamente gratificante, relativamente efímera, que requiere poco o ningún entrenamiento específico para su disfrute. El ocio basado en proyectos supone desempeños creativos en el corto plazo, razonablemente complicado, realizado una única vez u ocasional pero infrecuentemente, que se lleva a cabo en el tiempo libre, o en el tiempo liberado de obligaciones desagradables (Stebbins, 2011). El ocio serio conlleva un compromiso temporal discrecional (Stebbins, 2012) que incluye iniciativas o intencionalidades personales para asignar tiempo o reservar tiempo a las actividades de interés y además implican motivaciones, involucramientos y reconstrucciones de la identidad. 


\section{Objetivos}

1. Describir actividades de ocio que realizan los estudiantes de ingeniería y clasificarlas según la perspectiva del ocio serio.

2. Analizar la incidencia de variables socio-demográficas en las capacidades y acciones creativas de los estudiantes.

3. Analizar relaciones entre ocio y creatividad considerando capacidades y acciones creativas desarrolladas por los estudiantes.

4. Indagar relaciones entre creatividad y rendimiento académico teniendo en cuenta cantidad de materias rendidas y promedio general de notas.

5. Analizar relaciones entre creatividad, ocio y rendimiento a partir del estudio de casos de alumnos con elevado rendimiento académico y estudiantes potencialmente más creativos.

\section{Hipótesis}

1. Los puntajes medios en las mediciones de creatividad (capacidades y logros) varían en función de las variables sexo, edad, carrera elegida y actividad laboral.

2. Los alumnos que participan en actividades de ocio serio obtienen puntajes significativamente superiores en las mediciones de creatividad (capacidades y acciones creativas).

3. Los puntajes medios en las mediciones de creatividad (capacidades y logros) varían de manera significativa en función de la cantidad de actividades de ocio que realizan los estudiantes.

4. Las correlaciones entre rendimiento académico y creatividad (capacidades y logros) son positivas y moderadas

5. Los puntajes medios en las mediciones de creatividad (capacidades y logros) varían de manera significativa en función de la cantidad de materias aprobadas por los estudiantes.

6. Los estudiantes que logran aprobar materias tienen puntajes más altos en creatividad (capacidades y logros) respecto de los alumnos que no lograr aprobar materias.

\section{Métodos}

El diseño del estudio fue no experimental descriptivo correlacional según la clasificación de Hernández Sampieri, Fernández Collado y Baptista Lucio (1998). Se describieron variables referidas a creatividad, ocio y rendimiento académico luego, se pusieron a prueba hipótesis respecto de relaciones entre dichas variables.

\section{Participantes}

Participaron del estudio 132 alumnos de la Facultad de Ingeniería de la UNRC pertenecientes a la cohorte 2015. Si bien el listado de estudiantes inscriptos para iniciar carreras de ingeniería en el año 2015 totaliza 149 sujetos, fueron 132 quienes estaban presentes en el ámbito de la clase el día que se administraron los instrumentos. En este sentido, el muestreo fue no probabilístico y por oportunidad (Hernández Sampieri, Fernández Collado y Baptista Lucio, 1998). El 77\% de los participantes son de sexo masculino. Las edades oscilan entre 17 y 40 años, aunque la mayoría de los participantes (90\%) tiene entre 17 y 19 años. Participaron alumnos de las siguientes carreras de la Facultad de Ingeniería: Ingeniería Mecánica (40\%), Ingeniería Química (30\%); Ingeniería en Telecomunicaciones $(25 \%)$ e Ingeniería 
Electricista (5\%). El $28 \%$ de los participantes manifiesta desarrollar actividades laborales en paralelo a los estudios.

\section{Instrumentos}

Se recogieron datos en dos momentos. Primero, en el mes de febrero de 2015, cuando los estudiantes cursaban sus primeras clases en la universidad, se asistió a las aulas y se administraron dos cuestionarios (uno sobre aspectos sociodemográficos y otro sobre acciones creativas desarrolladas por los sujetos) y un test (que mide capacidades creativas). En un segundo momento, un año y medio después (en agosto de 2016), se tomaron datos de rendimiento académico (cantidad de materias aprobadas y promedio general de calificaciones) del Sistema de Información de Alumnos de la Universidad Nacional de Río Cuarto. Se describen a continuación las cuatro fuentes de información utilizadas:

1. Cuestionario socio-demográfico y de ocio. Todos los participantes respondieron un cuestionario elaborado para la presente investigación a fin de obtener datos respecto de variables socio-demográficas y actividades cotidianas (laborales y de ocio). Se les preguntó a los participantes qué actividades de tiempo libre realizaban y la dedicación horaria semanal a cada actividad. En el cuestionario se incluyeron actividades vinculadas al arte (plástica, literatura, música, etc.), la expresión corporal, la formación extracurricular (idiomas y computación, etc.), el voluntariado, la construcción de objetos e inventos y la realización de artesanías. También se incluyeron actividades como mirar televisión, escuchar música, usar redes sociales, juegos tecnológicos y vida social. Asimismo, el cuestionario cuenta con preguntas abiertas para incorporar otras actividades y oportunidades de hacer comentarios y valoraciones respecto de cada actividad.

2. Test sobre potencialidades creativas. Los potenciales creativos de las personas se evaluaron mediante el Test CREA (Corbalán et al., 2015) que utiliza como procedimiento de medida de la creatividad la capacidad del sujeto para elaborar preguntas. La prueba consta de tres láminas estímulo ( $A, B$ y $C$ ) a partir de las cuales los entrevistados deben formular la mayor cantidad de interrogantes posibles en un tiempo establecido. Dada las elevadas correlaciones entre las láminas del CREA obtenidas en estudios previos (Elisondo, 2015) y considerando las indicaciones del manual del instrumento, se administró la Lámina $A$. Numerosos estudios señalan las propiedades psicométricas del instrumento y de los avances logrados en la medición de capacidades creativas mediante el procedimiento de la generación de interrogantes (Clapham y King, 2010; Corbalán et al., 2015; López Martínez y Navarro Lozano, 2008; López Martínez y Brufau, 2010; Martínez Zaragoza, 2003). En el manual se detallan estudios psicométricos del instrumento: fiabilidad .87 en formas paralelas, análisis de validez convergente con pruebas de Guilford $(r=.61 ; r=.75 ; r=.59 ; r=.48)$, validez discriminante con pruebas de inteligencia $(r=.25 ; r=.20)$ y validez predictiva con puntuaciones de expertos respecto de tarea creativa (Corbalán et al., 2015). Martínez Zaragoza (2003) ha realizado un análisis de las propiedades psicométricas del CREA y ha concluido que el instrumento cumple con los estándares básicos de fiabilidad y validez exigibles a una prueba psicológica. En el estudio se observaron correlaciones elevadas entre las formas A, B y C del CREA y el factor de segundo orden subyacente a la Batería de Creatividad de Guilford ( $r=.750 ; r=.599 ; r=.784)$ y correlaciones muy bajas y no significativas entre el CREA y tres test de inteligencia (TEA, OTIS y TEI). Por su parte, Clapham y King (2010) examinan la confiabilidad y validez del CREA en una población de habla inglesa. Los resultados demostraron validez convergente del CREA con el TTCT de Torrance dimensión verbal $(r=.503)$ y dimensión figurativa $(r=.442)$. Los coeficientes de confiabilidad fueron elevados en la primera administración (.800) y en el re-test 
(.674). En un estudio de sistematización (Elisondo, 2015), se analizaron desempeños en el CREA de una muestra amplia de niños, adolescentes y adultos argentinos (4090 casos) y se confeccionaron baremos ajustados a las diferencias socio-demográficas observadas. Asimismo, se hallaron elevadas correlaciones entre los estímulos utilizados que aportan a los estudios de confiabilidad del instrumento (Niños Lámina $A$ y $B$ : $r=.769$, Lámina $B$ y $C: r=.823$, Lámina $A$ y $C$ : $r=.708$; Adolescentes Lámina $A$ y $B: r=.717$, Lámina $B$ y $C: r=.770$, Lámina $A$ y $C$ : $r=.611$; Adultos Lámina A y $B: r=.773$. Lámina $B$ y $C: r=.770$, Lámina A y C: $r=.806)$.

3. Cuestionario de Acciones Creativas. Las acciones creativas desarrolladas por los participantes previamente al ingreso en la universidad se evaluaron mediante el Cuestionario de Acciones Creativas -CAC- (Elisondo y Donolo, 2016). El instrumento utilizado consta de 40 ítems que evalúan la participación en 8 áreas de conocimiento: Literatura, Artes Plásticas, Ciencia y Tecnología, Expresión Corporal, Música, Artesanías, Participación Social y Creatividad Cotidiana. Los ítems refieren a acciones específicas en las áreas, por ejemplo, escribir un cuento, pintar un cuadro, y también reconocimientos de otros respecto de los desempeños, es decir, premios y distinciones obtenidas por las acciones desarrolladas. Asimismo, se incluyen ítems que valoran la participación de las personas en grupos y organizaciones vinculadas a cada área de conocimiento o actuación, enfatizando una perspectiva social de desarrollo de la creatividad donde las interacciones con otros resultan condicionantes de los procesos, tal como hemos observado en numerosas investigaciones. Los participantes deben responder en una escala Likert entre las siguientes opciones: 1 (nunca o casi nunca se ha hecho lo que se dice), 2 (si pocas veces lo ha hecho, 2 o 3 veces), 3 (si lo ha hecho varias veces, 4 o 5 veces), 4 (si lo hizo frecuentemente, 6 o 7 veces) y 5 (si en la mayoría de las veces que tuvo oportunidad lo hizo). El CAC ha demostrado una adecuada consistencia interna entre ítems y áreas definidas (alfa entre .72 y .83). La estructura factorial del CAC se corresponde con la propuesta inicial que incluye diferentes áreas de conocimiento para valorar desempeños creativos. Asimismo, se han observado interesantes relaciones entre el CAC y otras mediciones de la creatividad, se ha hallado una correlación significativa de .60 entre el Cuestionario de Actividades Creativas y el Biographical Inventory of Creative Behavior. Considerando estudios previos que indican relaciones entre ocio y creatividad (Aranguren y Irrazabal, 2012; Boyd Hegarty, 2009, Wolfradt y Pretz, 2001), se realizaron estudios de validez por criterio con grupos contrastados (personas que participan en actividades de ocio y personas que no participan), se ha observado en todas las áreas y en el puntaje total del CAC diferencias de media estadísticamente significativas a nivel de .000 entre los grupos. Las personas que participan en actividades de ocio obtuvieron puntajes medios significativamente superiores en todas las áreas del CAC y en el puntaje total (Elisondo y Donolo, 2016).

4. Datos sobre rendimiento académico tomados del Sistema de Información de Alumnos (SIAL). Para conocer respecto del rendimiento académico de los estudiantes - transcurrido un año y medio desde su ingreso-se recolectaron, con consentimiento de las autoridades de la Facultad, datos del SIAL. Específicamente, se analizó la cantidad de materias aprobadas por cada estudiante y el promedio general obtenido en los exámenes finales. Este seguimiento permitió identificar tres grupos de estudiantes: el grupo que aprobó al menos 1 asignatura $(\mathrm{N}=82)$, otro grupo que no aprobó ninguna en el transcurso del año y medio de cursado y que presuntamente abandonó sus estudios $(\mathrm{N}=37)$ y un tercer grupo $(\mathrm{N}=13)$ que ya no se encontraba en el SIAL, posiblemente por no haber culminado estudios de nivel medio durante 2015 y no haber completado el trámite de inscripción en la Facultad, no siendo entonces alumnos efectivos de la Facultad de Ingeniería. 


\section{Procedimiento}

Los dos cuestionarios mencionados y el Test CREA se aplicaron durante las actividades de ingreso a la vida universitaria, es decir en febrero de 2015. Luego se recolectaron datos respecto de rendimiento académico al finalizar el primer cuatrimestre de cursado correspondiente al segundo año de estudio, es decir en agosto de 2016.

Los test y cuestionarios fueron tomados de manera grupal y en formato papel y lápiz. Se cuenta con el consentimiento informado de los participantes para la realización de la investigación y la publicación de resultados preservando la confidencialidad de los datos. También se cuenta con consentimiento de las autoridades de la facultad para el manejo de datos respecto de rendimiento académico de los estudiantes.

\section{Análisis de datos}

Se realizaron análisis estadísticos univariados y bivariados con el programa SPSS Versión 20: estudios de frecuencias, medias, desviaciones estándar y pruebas de diferencia de media. Se pusieron a prueba las hipótesis mediante análisis de diferencia de media (prueba $t$ y ANOVA) y estudio de correlaciones mediante coeficiente de Spearman.

\section{Resultados}

En primer lugar, se analizan relaciones entre creatividad y ocio en estudiantes ingresantes en 2015 en carreras de Ingeniería de la UNRC, se consideran datos recolectados durante las Actividades de Ingreso a la Vida Universitaria. Luego, se estudian vinculaciones entre ocio, creatividad y rendimiento en el grupo de alumnos que continúan sus estudios en 2016 y han aprobado al menos una asignatura del plan de estudios. Posteriormente, se comparan mediciones de creatividad y ocio en dos grupos: quienes han rendido al menos una materia y quienes no lo han logrado o ya no se encuentran en el SIAL. Por último, se analizan casos de estudiante potencialmente más creativos y alumnos con elevado rendimiento académico.

\section{Creatividad y ocio en estudiantes ingresantes}

Considerando los resultados de los 132 participantes en el CREA, se observó que el puntaje directo mínimo fue 3 y el máximo 21, la media de 10.20 y la desviación estándar de 3.67. Los análisis indican que la mayoría de los estudiantes encuestados disponen de capacidades creativas medias $(N=98)$ y altas $(N=15)$ según los criterios interpretativos del Test CREA. Se realizaron estudios de diferencia de media (prueba $t$ y ANOVA) en las mediciones de creatividad según sexo, edad, carrera elegida y actividad laboral. Solo se observaron diferencias estadísticamente significativas en la variable sexo; las mujeres obtuvieron puntajes medios superiores que los varones en el puntaje total del CAC y en las áreas Literatura, Actuación, Artesanías y Participación Social.

Respecto de las actividades de tiempo libre, la mayoría de los participantes dice realizar salidas y visitas con amigos, familiares y la pareja $(\mathrm{N}=132)$; interactuar con tecnologías en juegos, redes sociales, etc. $(\mathrm{N}=130)$ y mirar TV y escuchar música $(\mathrm{N}=129)$. Muchos estudiantes dicen realizar actividad física y deportiva en su tiempo libre $(\mathrm{N}=114)$. Otras actividades que se mencionaron con menor frecuencia fueron: actividades vinculadas con la música, específicamente ejecutar un instrumento musical $(\mathrm{N}=46)$; estudiar idiomas en su tiempo libre $(\mathrm{N}=28)$; realizar inventos y creaciones 
originales $(\mathrm{N}=24)$, realizar artesanías $(\mathrm{N}=20)$ y fotografía $(\mathrm{N}=16)$. Quince estudiantes participan en voluntariados y en actividades en ONGs, 14 en actividades literarias y se dedican a las artes plásticas y visuales 13 estudiantes de la muestra. Ocho estudiantes realizan danzas y cuatro expresión corporal o actuación. Diecisiete estudiantes manifestaron realizar otras actividades además de las incluidas en el cuestionario, como por ejemplo (tareas domésticas, arreglos en el hogar, jardinería, etc.). Considerando las respuestas de los estudiantes respecto de las actividades que desarrollaban y la cantidad de horas que les dedicaban, se identificaron a los estudiantes que realizan ocio serio $(\mathrm{N}=79)$, es decir que participan de manera sistemática y comprometida en alguna actividad artística, cultural, inventiva, social o comunitaria (Stebbins, 2012). Se observó que 56 estudiantes desarrollan una actividad de ocio serio, 16 alumnos participan en dos actividades y 7 en más de dos actividades.

Se compararon grupos de alumnos según participen o no en actividades de ocio serio en todas las mediciones de creatividad (Test CREA para capacidades y CAC para acciones creativas). Se observó que las personas que participan en actividades de ocio serio obtienen puntajes medios significativamente superiores en el CREA, el total del CAC y en las áreas artes plásticas, actuación y música (Ver Tabla 1).

\begin{tabular}{|c|c|c|c|c|c|c|}
\hline & Ocio Serio & $\mathbf{N}$ & $M$ & DE & $t$ & $p$ \\
\hline \multirow[t]{2}{*}{ CREA } & No Participa & 53 & 9.32 & 3.47 & -2.41 & 017 \\
\hline & Participa & 79 & 10.87 & 3.70 & & \\
\hline \multirow[t]{2}{*}{$\overline{C A C}$} & No Participa & 53 & 68.63 & 14.64 & -3.66 & .000 \\
\hline & Participa & 79 & 80.75 & 20.69 & & \\
\hline \multirow[t]{2}{*}{ Lit } & No Participa & 53 & 7.28 & 1.97 & -1.55 & .123 \\
\hline & Participa & 79 & 7.93 & 2.59 & & \\
\hline \multirow[t]{2}{*}{ Artes } & No Participa & 53 & 7.41 & 2.45 & -2.12 & .036 \\
\hline & Participa & 79 & 8.88 & 4.62 & & \\
\hline \multirow[t]{2}{*}{ Actua } & No Participa & 53 & 6.64 & 2.34 & -2.50 & .014 \\
\hline & Participa & 79 & 8.16 & 3.98 & & \\
\hline \multirow[t]{2}{*}{ Música } & No Participa & 53 & 7.11 & 5.85 & -2.90 & .004 \\
\hline & Participa & 79 & 9.93 & 5.15 & & \\
\hline \multirow[t]{2}{*}{ Artesa } & No Participa & 53 & 8.50 & 2.93 & $\begin{array}{l}-1.63 \\
\end{array}$ & .104 \\
\hline & Participa & 79 & 9.49 & 3.65 & & \\
\hline \multirow[t]{2}{*}{ P. Social } & No Participa & 53 & 9.56 & 3.18 & -.95 & .340 \\
\hline & Participa & 79 & 10.17 & 3.84 & & \\
\hline Ciencia y & No Participa & 53 & 7.35 & 2.14 & -1.44 & .151 \\
\hline Tecnología & Participa & 79 & 7.97 & 2.56 & & \\
\hline \multirow[t]{2}{*}{ Cotidiana } & No Participa & 53 & 15.03 & 4.43 & -1.77 & .079 \\
\hline & Participa & 79 & 18.18 & 12.42 & & \\
\hline \multicolumn{7}{|c|}{$\begin{array}{l}\text { CREA: Test Crea; CAC=Cuestionario de Acciones Creativas. Puntaje total; Lit=Área literatura del CAC; Arte=Área Artes } \\
\text { Plásticas del CAC; Actúa=Área Actuación y Expresión Corporal del CAC; Música=Área Música del CAC; Artesa=Área } \\
\text { Artesanías del CAC; P. Social=Área Participación Social del CAC; Ciencia=Área Ciencia y Tecnología del CAC; } \\
\text { Cotidiana=Área Creatividad cotidiana del CAC. }\end{array}$} \\
\hline
\end{tabular}

También se estudiaron diferencias de media en las mediciones de creatividad considerando la cantidad de actividades de ocio serio que realizaban los estudiantes. En la Tabla 2 se visualiza que en todas las mediciones, excepto en creatividad cotidiana, los puntajes medios ascienden conforme aumenta la cantidad de actividades de ocio serio que realizan los estudiantes. Se observaron diferencias significativas según ANOVA en el CREA, el total del CAC y en las áreas literatura, artes plásticas, actuación y música considerando cantidad de actividades de ocio que los participantes realizan. 
Tabla 2. Puntajes medios, desviaciones estándar y ANOVA prueba t en mediciones de creatividad (CREA y CAC) según cantidad de actividades de ocio en las que participan.

\begin{tabular}{|c|c|c|c|c|c|c|c|c|c|c|c|}
\hline \multirow{4}{*}{$\frac{\text { Ocio Serio }}{\text { No participa }}$} & & CAC & CREA & Lit & Arte & Actua & Música & Artesa & P. Soc. & Ciencia & Cotidiana \\
\hline & $M$ & 68.63 & 9.32 & 7.28 & 7.41 & 6.64 & 7.11 & 8.50 & 9.56 & 7.35 & 15.03 \\
\hline & $\mathrm{N}$ & 53 & 53 & 53 & 53 & 53 & 53 & 53 & 53 & 53 & 53 \\
\hline & DE & 14.64 & 3.47 & 1.97 & 2.45 & 2.34 & 5.85 & 2.93 & 3.18 & 2.14 & 4.43 \\
\hline \multirow[t]{3}{*}{ Una actividad } & M & 78.44 & 10.25 & 7.33 & 8.41 & 7.44 & 9.53 & 9.25 & 9.78 & 7.78 & 18.89 \\
\hline & $\mathrm{N}$ & 56 & 56 & 56 & 56 & 56 & 56 & 56 & 56 & 56 & 56 \\
\hline & DE & 21.03 & 3.28 & 2.21 & 5.00 & 3.52 & 4.79 & 3.81 & 3.55 & 2.29 & 14.52 \\
\hline \multirow[t]{3}{*}{ Dos actividades } & M & 80.50 & 12.00 & 8.68 & 8.81 & 8.93 & 9.56 & 10.00 & 10.43 & 8.25 & 15.81 \\
\hline & $\mathrm{N}$ & 16 & 16 & 16 & 16 & 16 & 16 & 16 & 16 & 16 & 16 \\
\hline & DE & 15.32 & 3.79 & 2.05 & 2.13 & 3.92 & 5.36 & 3.48 & 3.48 & 2.79 & 3.41 \\
\hline \multirow{5}{*}{$\begin{array}{l}\text { Más de dos } \\
\text { actividades }\end{array}$} & $M$ & 99.85 & 13.29 & 11.00 & 12.85 & 12.14 & 14.00 & 10.28 & 12.71 & 8.85 & 18.00 \\
\hline & $\mathrm{N}$ & 7 & 7 & 7 & 7 & 7 & 7 & 7 & 7 & 7 & 7 \\
\hline & $\mathrm{DE}$ & 21.24 & 5.43 & 4.04 & 4.14 & 5.42 & 6.40 & 2.92 & 6.07 & 4.01 & 4.96 \\
\hline & ANOVA & 7.614 & 4.20 & 7.276 & 4.399 & 6.910 & 4.313 & 4.313 & 1.747 & 1.192 & 1.428 \\
\hline & $p$ & .000 & .007 & .000 & .006 & .000 & .006 & .303 & 161 & .316 & .238 \\
\hline
\end{tabular}

CREA: Test Crea; CAC= Cuestionario de Acciones Creativas. Puntaje total; Lit= Área literatura del CAC; Arte= Área Artes Plásticas del CAC; Actúa=Área Actuación y Expresión Corporal del CAC; Música= Área Música del CAC; Artesa= Área Artesanías del CAC; P. Social=Área Participación Social del CAC; Ciencia= Área Ciencia y Tecnología del CAC; Cotidiana= Área Creatividad cotidiana del CAC

\section{Creatividad, ocio y rendimiento: estudiantes que aprobaron al menos 1 asignatura}

Según datos tomados del Sistema de Información de Alumnos, de los 132 estudiantes que participaron de la investigación en febrero de 2015, 82 lograron aprobar al menos 1 asignatura en el marco de la carrera que cursan. El 76\% son varones. Las edades oscilan entre 18 y 30 años, aunque la mayoría $85 \%$ tiene 18 o 19 años. Respecto de las carreras que cursan, el grupo se distribuye de la siguiente manera: Ingeniería Mecánica (28\%), Ingeniería Química (45\%); Ingeniería en Telecomunicaciones (22\%) e Ingeniería Electricista (5\%). Veinte estudiantes (24\%) realizan actividades laborales. No se observaron relaciones elevadas ni significativas entre creatividad (capacidades y acciones) y rendimiento académico considerando el promedio general de notas (Ver Tabla 3).

Tabla 3. Correlaciones según coeficiente de Spearman entre mediciones de creatividad y rendimiento académico (promedio general).

\begin{tabular}{|c|c|c|c|c|c|c|c|c|c|c|c|}
\hline & & CAC & RA & Lit & Arte & Música & Actuación & Ciencia & Artesa & Cotidiana & P.Social \\
\hline \multirow[t]{2}{*}{ CREA } & Rs & $.370^{* *}$ & .024 & .180 & .094 & $.299^{* *}$ & $.245^{*}$ & $.259^{*}$ & -.052 & $.227^{*}$ & $.293^{* *}$ \\
\hline & $p$ & .001 & .830 & .106 & .399 & .006 & .027 & .019 & 644 & .040 & .008 \\
\hline \multirow[t]{2}{*}{ CAC } & rs & & -009 & $.539^{* *}$ & $.319^{* *}$ & $.459^{* *}$ & $.411^{* *}$ & $.471^{* *}$ & $.364^{* *}$ & $.795^{* *}$ & $.678^{* *}$ \\
\hline & $p$ & & .937 & .000 & .003 & .000 & .000 & .000 & .001 & .000 & .000 \\
\hline \multirow[t]{2}{*}{ RA } & rs & & & -.070 & .061 & -.036 & -.157 & .106 & $.226^{*}$ & -.027 & .006 \\
\hline & $p$ & & & .530 & .585 & .749 & .158 & .341 & .041 & .806 & .959 \\
\hline \multirow[t]{2}{*}{ Lit } & rs & & & & $.253^{*}$ & -.027 & $.479^{* *}$ & $.396^{* *}$ & $.245^{\star}$ & $.283^{* *}$ & $.345^{* *}$ \\
\hline & $p$ & & & & .022 & .806 & .000 & .000 & .027 & .010 & .002 \\
\hline \multirow[t]{2}{*}{ Arte } & rs & & & & & .003 & .097 & $.314^{* *}$ & $.292^{\star *}$ & .031 & .201 \\
\hline & $p$ & & & & & .980 & .385 & .004 & .008 & .783 & .071 \\
\hline \multirow[t]{2}{*}{ Música } & rs & & & & & & .105 & .038 & -.089 & $.260^{*}$ & .141 \\
\hline & $p$ & & & & & & .349 & .733 & .425 & .018 & .206 \\
\hline \multirow[t]{2}{*}{ Actúa } & rs & & & & & & & $.333^{* *}$ & .165 & .008 & $.450^{* *}$ \\
\hline & $p$ & & & & & & & .002 & .138 & .941 & .000 \\
\hline \multirow[t]{2}{*}{ Ciencia } & rs & & & & & & & & .197 & .140 & $.432^{* *}$ \\
\hline & $p$ & & & & & & & & .076 & .210 & .000 \\
\hline \multirow{2}{*}{ Artesa } & rs & & & & & & & & & .109 & $.267^{*}$ \\
\hline & $p$ & & & & & & & & & .329 & .015 \\
\hline \multirow[t]{2}{*}{ cotidiana } & rs & & & & & & & & & & $.395^{* *}$ \\
\hline & $p$ & & & & & & & & & & .000 \\
\hline
\end{tabular}

CREA: Test Crea; CAC=Cuestionario de Acciones Creativas. Puntaje total; Lit=Área literatura del CAC; Arte=Área Artes Plásticas del CAC; Actúa=Área Actuación y Expresión Corporal del CAC; Música=Área Música del CAC; Artesa= Área Artesanías del CAC; P. Social=Área Participación Social del CAC; Ciencia= Área Ciencia y Tecnología del CAC; Cotidiana= Área Creatividad cotidiana del CAC. 
Sin embargo, se observó que los estudiantes que habían aprobado más materias (entre 8 y 10) tenían puntajes medios significativamente superiores en la escala relativa a Ciencia y Tecnología del CAC respecto de quienes habían rendido entre 1 y 3 materias o entre 4 y 7 (Ver tabla 4 ).

Tabla 4. Puntajes medios, desviaciones estándar y ANOVA en mediciones de creatividad (CREA y CAC) según cantidad de materias aprobadas. Tabla de elaboración propia.

\section{Materias}

$P$. aprobadas CREA CAC Lit. Arte MúsicaActúaCienciaArtesaCotidianaSocial

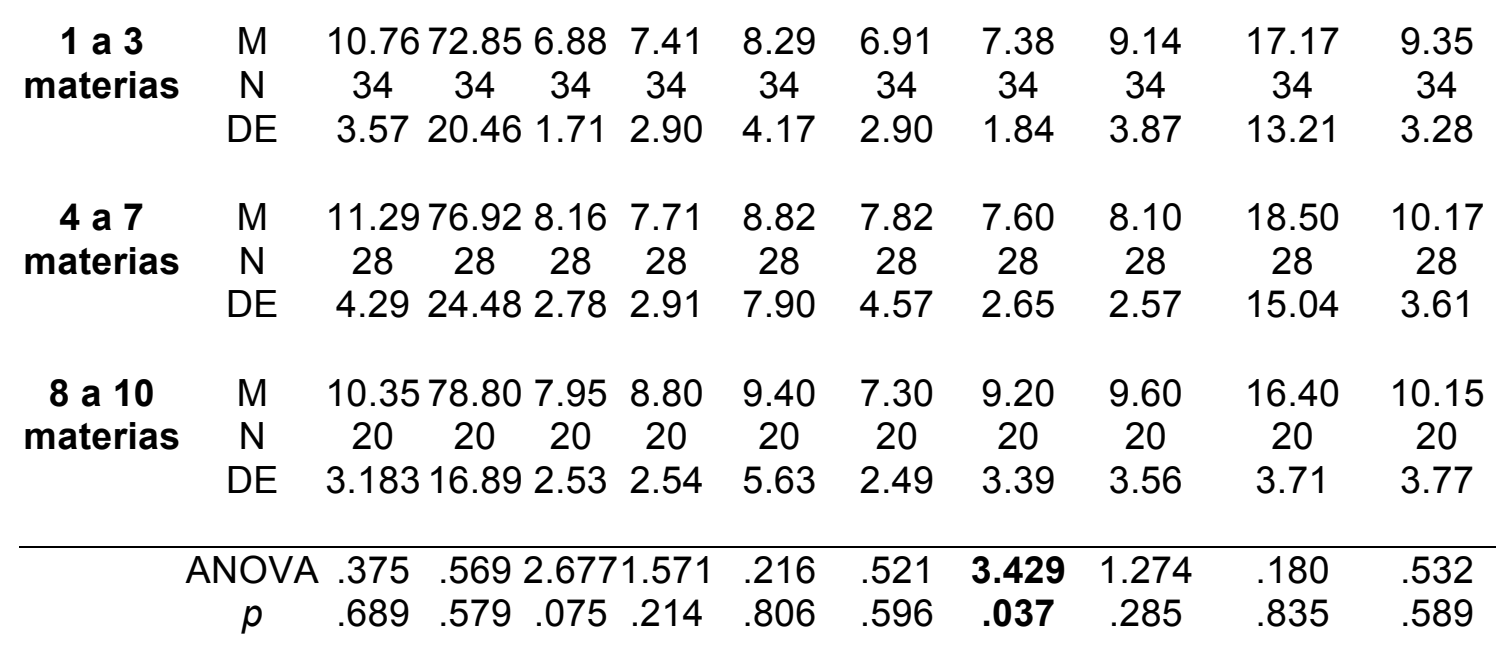

CREA: Test Crea; $C A C=$ Cuestionario de Acciones Creativas. Puntaje total; Lit= Área literatura del CAC; Arte= Área Artes Plásticas del CAC; Actúa=Área Actuación y Expresión Corporal del CAC; Música= Área Música del CAC; Artesa= Área Artesanías del CAC; P. Social=Área Participación Social del CAC; Ciencia= Área Ciencia y Tecnología del CAC; Cotidiana= Área Creatividad cotidiana del CAC

En general, se observa que los estudiantes que aprobaron más materias tienen puntajes más elevados que el resto en las mediciones de creatividad (capacidades y acciones creativas). En grupos con bajo rendimiento (promedio general entre 2 y 4.99), rendimiento medio (promedio general entre 5 y 7.99) y rendimiento alto (promedio general de 8 a 9.25), no se observaron diferencias significativas en los puntajes medios en las mediciones de creatividad. También se analizaron los promedios de los alumnos según participación en actividades de ocio serio, no observándose diferencias entre quienes participan y quienes no lo hacen. 


\section{Creatividad en estudiantes que aprobaron y que no aprobaron materias}

Luego de recolectar datos respecto del rendimiento académico de los estudiantes en 2016, se observó que 37 estudiantes no aprobaron ninguna materia habiendo transcurrido un año y medio desde el ingreso, mientras que 13 ya no se encontraban en el sistema probablemente por no haber culminado el nivel medio en 2015.

Considerando los datos disponibles en las mediciones de creatividad y ocio, se compararon ambos grupos (estudiantes que aprobaron y estudiantes que no aprobaron materias) y se observó que los estudiantes que aprobaron materias habían obtenido puntajes significativamente más altos en capacidades creativas según el Test CREA (Ver tabla 5).

\begin{tabular}{|c|c|c|c|c|c|c|}
\hline & Grupos & $\mathbf{N}$ & $M$ & $\mathrm{DE}$ & $t$ & $p$ \\
\hline \multirow[t]{2}{*}{$\overline{\text { CREA }}$} & $\mathrm{G} 2$ & 50 & 9.16 & 3.37 & -2.60 & .010 \\
\hline & G1 & 82 & 10.84 & 3.72 & & \\
\hline \multirow[t]{2}{*}{ CAC } & G2 & 50 & 76.66 & 16.43 & .312 & .755 \\
\hline & $\mathrm{G} 1$ & 82 & 75.57 & 20.99 & & \\
\hline \multirow[t]{2}{*}{ Lit. } & G2 & 50 & 7.74 & 2.38 & .36 & .718 \\
\hline & G1 & 82 & 7.58 & 2.38 & & \\
\hline \multirow[t]{2}{*}{ Arte } & $\overline{\mathrm{G} 2}$ & 50 & 8.94 & 5.28 & 1.53 & .127 \\
\hline & G1 & 82 & 7.85 & 2.84 & & \\
\hline \multirow[t]{2}{*}{ Actúa } & G2 & 50 & 7.98 & 3.53 & 1.058 & .292 \\
\hline & G1 & 82 & 7.31 & 3.47 & & \\
\hline \multirow[t]{2}{*}{ Música } & $\overline{\mathrm{G} 2}$ & 50 & 8.84 & 4.97 & .095 & .924 \\
\hline & G1 & 82 & 8.74 & 5.96 & & \\
\hline \multirow[t]{2}{*}{ Artesa } & G2 & 50 & 9.38 & 3.40 & .781 & .436 \\
\hline & G1 & 82 & 8.90 & 3.41 & & \\
\hline \multirow[t]{2}{*}{ P. Social } & $\mathrm{G} 2$ & 50 & 10.22 & 3.73 & .606 & .545 \\
\hline & G1 & 82 & 9.82 & 3.49 & & \\
\hline \multirow[t]{2}{*}{ Ciencia } & $\overline{\mathrm{G} 2}$ & 50 & 7.36 & 1.80 & -1.279 & .203 \\
\hline & G1 & 82 & 7.90 & 2.64 & & \\
\hline \multirow[t]{2}{*}{ Cotidiana } & G2 & 50 & 16.20 & 4.90 & -.681 & .497 \\
\hline & G1 & 82 & 17.43 & 12.26 & & \\
\hline
\end{tabular}

CREA: Test Crea; CAC=Cuestionario de Acciones Creativas. Puntaje total; Lit=Área literatura del CAC; Arte=Área Artes Plásticas del CAC; Actúa=Área Actuación y Expresión Corporal del CAC; Música= Área Música del CAC; Artesa= Área Artesanías del CAC; P. Social=Área Participación Social del CAC; Ciencia=Área Ciencia y Tecnología del CAC; Cotidiana=Área Creatividad cotidiana del CAC.

\section{Análisis de casos}

Considerando la muestra inicial $(\mathrm{N}=132)$, se analizaron casos de estudiantes con puntajes altos en el Test CREA, seleccionándose los 10 estudiantes con mejores desempeños en el test. Se observó que solo 2 de estos estudiantes no lograron rendir ninguna materia. Los demás son estudiantes regulares y han rendido entre 4 y 7 materias con promedios entre 4 y 7,90. Ninguno de los estudiantes con puntajes altos en el CREA tiene promedios altos (8 o más). Ocho de los estudiantes con elevadas potencialidades creativas realizan actividades de ocio serio, 3 participan en una actividad, igual número en dos actividades, un estudiante participa en tres actividades y el restante se desempeña en 5 actividades. En el total del CAC, cinco estudiantes obtuvieron puntajes que se ubican en el segundo cuartil, e igual cantidad en el tercer cuartil. Es decir, los estudiantes potencialmente más creativos obtienen puntajes medios y altos en el cuestionario de acciones creativas.

Considerando los estudiantes que habían rendido al menos una materia $(N=87)$, se seleccionaron los 10 casos de mejor rendimiento teniendo en cuenta el promedio general y la cantidad de materias aprobadas. Se eligieron aquellos casos que tenían promedios más altos y habían rendido al menos 5 materias. Los diez alumnos de 
mejor rendimiento tienen puntajes en el CREA correspondientes a creatividad media. Seis de los diez estudiantes con mejor rendimiento hacen actividades de ocio serio, 4 alumnos participan en 1 actividad y 2 alumnos en dos actividades. Tres estudiantes obtuvieron puntajes en el CAC que se ubican en el primer cuartil, cinco en el segundo $\mathrm{y}$ dos en el tercero.

Ningún estudiante forma parte de los dos grupos definidos (potencialmente más creatividad y alumnos de más alto rendimiento). Es decir, ningún estudiante puede considerarse como potencialmente creativo y de alto rendimiento académico.

\section{Discusión}

Entre los resultados más importantes se observa que los estudiantes encuestados disponen, en general, de potencialidades creativas medias y altas. Además se han desempeñado en acciones creativas en diferentes campos de conocimiento. Se visualizaron diferencias en las mediciones de acciones creativas según sexo, se corrobora la Hipótesis 1 solo para esta variable, dato que se corresponde con otras investigaciones similares (Aranguren y Irazábal, 2012; Elisondo y Donolo, 2016).

Se ha corroborado la Hipótesis 2, los alumnos que participan en actividades de ocio serio obtienen puntajes significativamente superiores en las mediciones de creatividad, en el CREA, el CAC total y las áreas arte, actuación, música y creatividad cotidiana. Al igual que en estudios anteriores (Elisondo y Donolo, 2016; Wolfradt y Pretz, 2001) y corroborando la Hipótesis 3 , se ha observado que las personas que participan en más actividades de ocio obtienen mejores resultados en las puntaciones de creatividad. Las personas que más participan en actividades de ocio, y especialmente en propuestas de ocio serio, serían más abiertas a la experiencia. Numerosos estudios (ChamorroPremuzic y Furnham, 2003; Hunter et al., 2016) han aportado evidencias respecto de las relaciones entre creatividad y el rasgo de personalidad apertura a la experiencia. Las personas creativas demuestran un especial interés por el entorno y por participar en experiencias diversas, como lo son por ejemplo las actividades de ocio que han sido objeto de investigación en el presente estudio. Asimismo, la participación en diversas actividades de ocio puede vincularse con el rasgo de personalidad Extraversión, rasgo que en diversas investigaciones ha correlacionado con las mediciones de creatividad (McKay, Karwowski y Kaufman, 2017) En este sentido, la participación en diversas actividades de ocio podría relacionarse con la sociabilidad, gusto y facilidad para las relaciones sociales, despreocupación y preferencia por la acción frente a la reflexión. Los estudiantes más creativos, tanto considerando capacidades como acciones creativas, son aquellos que participan en actividades de ocio serio. Asimismo, se observa que quienes más participan obtienen puntajes más elevados en las mediciones de creatividad.

Si bien no se han observado relaciones entre rendimiento, ocio y creatividad es decir, no se corrobora la Hipótesis 4, los hallazgos obtenidos se orientan a considerar la relevancia de promover en los estudiantes la participación en actividades de ocio serio en tanto oportunidades para el desarrollo de acciones creativas. Especialmente considerando que quienes habían desarrollado mayor cantidad de acciones creativas en el área ciencias y tecnologías han logrado mejores desempeños teniendo en cuenta la cantidad de materias aprobadas. Se corrobora la Hipótesis 5 solo para el área Ciencia y Tecnología del CAC. Asimismo, es relevante promover la participación en ocio serio atendiendo a los demostrados impactos positivos en la salud de este tipo de actividades (Mannell, 2007; Newman, Tay y Diener, 2014). 
Tal como se ha planteado en estudios anteriores (Chamorro-Premuzic y Furnham, 2003; Xiaoxia Ai, 1999), las relaciones entre creatividad y rendimiento parecen ser complejas y depender de los grupos y los instrumentos que se utilizan. No es posible afirmar que los alumnos más creativos tienen los mejores rendimientos académicos y tampoco puede aseverarse lo contrario. Los estudios de casos indican que los más creativos tienen rendimientos medios y que los estudiantes de mejor rendimiento disponen de capacidades creativas medias. Al igual que sucede con las relaciones entre creatividad e inteligencia, un mínimo de inteligencia es necesaria para la creatividad (Karwowski et al., 2016; Corbalán et al., 2015), cierta base creativa parece ser indispensable para tener buenos desempeños académicos. De hecho, no se observan casos de alumnos de rendimiento alto y creatividad baja.

La creatividad también parece ser un recurso importante para resolver problemas propios del oficio de ser estudiante. Se ha observado que los alumnos que han logrado rendir materias mostraron mejores desempeños en el CREA, corroborando solo en esta medición la Hipótesis 6 . La mayoría de los alumnos más creativos continúan sus estudios y ha logrado rendir entre 4 y 7 materias.

Interesantes líneas de investigación se abren al pensar las relaciones entre creatividad, continuidad y regularidad en los estudios universitarios. Considerar diversos grupos y diversas metodologías y perspectivas de análisis respecto de estas posibles relaciones es un desafío para estudios futuros. Acordamos con Spuzic y colaboradores (2016) en la necesidad de promover pensamientos críticos y creativos en la educación de los futuros ingenieros. En este sentido, es relevante como futura línea de investigación e intervención educativa generar espacios educativos orientados al desarrollo de pensamientos críticos y acciones creativas en actividades curriculares y extracurriculares en la Universidad. Asimismo, como futuras líneas de investigación se propone analizar a partir de estudios longitudinales las trayectorias educativas del grupo investigado. También es interesante realizar estudios de triangulación que integren metodologías cualitativas como entrevistas y grupos focales. De mismo modo, es relevante llevar a cabo estudios comparativos donde se incluyan grupos de otras facultadles y campos de conocimiento, a los efectos de analizar particularidades de las relaciones entre creatividad, ocio y rendimiento en grupos diversos. Concretamente se propone en futuros estudios realizar comparaciones entre estudiantes de ingeniería y alumnos de otras carreras y universidades. Asimismo, resulta interesa analizar, desde la perspectiva de la creatividad, contextos de enseñanza y aprendizaje en carrera de ingeniera para identificar condicionantes de los procesos creativos. Futuros estudios también pueden orientarse al análisis del impacto del ocio y la creatividad en la salud y el bienestar de los alumnos. El análisis de relaciones entre ocio, creatividad y rendimiento académico a lo largo de la formación de grado también es una temática de relevancia. Indagar estas variables con relación a la inserción laboral y el desempeño profesional es una línea promisoria especialmente para los laboratorios de monitoreo de graduados. Desde la perspectiva cualitativa, futuros estudios pueden interpretar sentidos y significados construidos por los estudiantes respecto del ocio, las actividades extracurriculares en la universidad, la creatividad y las trayectorias académicas.

El estudio presenta limitaciones en cuanto al tipo de muestreo utilizado, el mismo no permite realizar generalizaciones a otros contextos y situaciones. Respecto de los instrumentos, el cuestionario referido a ocio se encuentra en proceso de validación por lo tanto, no se disponen de indicadores técnicos respecto de su calidad. También resulta una limitante la consideración de sólo dos dimensiones del rendimiento académico (promedio general y materias aprobadas), en futuros estudios es necesario complejizar la operacionalización de esta variable. 
En definitiva, de acuerdo con los resultados obtenidos y las interpretaciones elaboradas al respecto, entendemos que los aportes brindados en este estudio constituyen insumos valiosos para pensar, tanto desde los ámbitos académicos como familiares, en una educación y formación de los niños y jóvenes orientada a promover la diversidad de experiencias, el compromiso con instancias de ocio serio y el emprendimiento de actividades creativas diversas. Es indispensable construir nuevos espacios de formación dentro y fuera de los contextos formales que consideren experiencias diferentes y entornos heterogéneos donde construir conocimientos.

\section{Referencias}

Alencar, EMLS., \& Fleith, DS. (2008). Criatividade pessoal: fatores facilitadores e inibidores segundo estudantes de engenharia. Magis, Revista Internacional de Investigación en Educación, $1,113-126$.

Alonso, R., Martínez, M. y Martín, P. (2015). Creatividad, atención y rendimiento académico en alumnado de conservatorio profesional. International Journal of Developmental and Educational Psychology, 1, 473-482.

Aranguren, M. e Irazábal, N. (2012). Diseño de una Escala para la evaluación del Comportamiento Creativo en diferentes dominios. Ciencias Psicológicas, 6(1), 29-41.

Balgiu, B., \& Adir, A. (2014). Creativity Tasks and Academic Achievement. A Study on Romanian Politehnica Undergraduate Students. Procedia - Social and Behavioral Sciences, 116, 924-928.

Cabeza, MC. (2017). Ocio y recreación para el empoderamiento de la ciudadanía. En Lema, R. y Monteagudo, M. (eds.) Espacios de ocio y recreación para la construcción de ciudadanía. (pp. 25-42). Universidad de Deusto.

Chamorro-Premuzic, T., \& Furnham, A. (2003) Personality predicts academic performance: Evidence from two longitudinal university samples. Journal of Research in Personality, 37, 319-338.

Chiecher, A. (en prensa) Estudiantes de ingeniería. Perfiles asociados con trayectorias de logro. En Panaia, M. (Coord.) De la formación al empleo. El desafío de la innovación. Buenos Aires: Miño y Dávila.

Clapham, M. y King, W. (2010). Psychometric Characteristics of the CREA in an English Speaking Population. Anales de Psicología, 26(2), 206-211.

Corbalán, J., Martínez, F., Donolo, D., Alonso, C., Tejerina, M. y Limiñana, M. (2015). CREA. Inteligencia Creativa. Una medida Cognitiva de la Creatividad. Madrid: TEA Ediciones. Second Edition.

Cropley, D. (2015). Creativity in engineering. En GE. Corazza and S. Agnoli (Eds.), Multidisciplinary Contributions to the Science of Creative Thinking, (pp. 155-173). London: Springer.

Csikszentmihalyi, M. (2001) Ocio y creatividad en el desarrollo humano. En Csikszentmihalyi, M; Cuenca, M. Buarque, C. y Trigo C. (2001). Ocio y desarrollo: potencialidades del ocio para el desarrollo humano, (pp. 17-32). Bilbao: Universidad de Deusto.
Elizalde, R., Gomes, C. L., Bahia, M. C., y Lacerda, L. L. (2015). Contribución del ocio para la sustentabilidad y los desafíos ambientales del presente. Estudios y perspectivas en turismo, 24(3), 493-511.

Elisondo, R. (2015). Evaluación de la creatividad. Análisis de variables alternativas relacionadas con la forma y el contenido de las respuestas en el CREA. Tesis de Doctorado. Universidad de Murcia.

Elisondo R. y Donolo, D. (2015). Creatividad en la Universidad. Tenerife: Cuadernos de Educación. Sociedad Latina de Comunicación Social. Universidad de la Laguna.

Elisondo, R., y Donolo, D. (2016). Construcción y análisis de las propiedades psicométricas del Cuestionario de Acciones Creativas en población argentina. PSIENCIA. Revista Latinoamericana de Ciencia Psicológica, 8, 1-21.

Fuentes, S., Velázquez, M., Abarca, M., Covarrubias, M. y Figueroa, M. (2015). Análisis de la relación entre creatividad, inteligencia múltiple y rendimiento académico de estudiantes de un Bachillerato en Artes del Estado de Colima, México Revista Internacional de Pedagogía y Currículo, 2(1), 15-25.

Hegarty, C. (2009). The Value and Meaning of Creative Leisure. Psychology of Aesthetics, Creativity and the Arts, 3(1), 10-13.

Hernández Sampieri, R., Fernández Collado, C. y Baptista Lucio, P. (1998) Metodología de la investigación. México: McGrawHill.

Gajda, A. (2016). The relationship between school achievement and creativityat different educational stages. Thinking Skills and Creativity, 19, 246-259.

Gómez, M. y Lago, C. (2017). Actividades extra-académicas, formación profesional y creatividad. En Elisondo, R. y Melgar, M. Creatividad y Universidad. Estudios más allá de los muros. (pp. 17-58). Malaga: Eumed.

Hunter, J., Abraham, E., Hunter, A., Goldberg, L., \& Eastwood, J. (2016). Personality and boredom proneness in the prediction ofcreativity and curiosity. Thinking Skills and Creativity, 22, 48-57.

Karwowski, M., Dul, J., Gralewski, J., Jauk, E., Jankowska, D., Gajda, A., Chruszczewski, M., \& Benedek, M. (2016). Is creativity without intelligence possible? A Necessary Condition Analysis. Intelligence, 57, 105-117. 
Limiñana, M., Bordoy, M., Juste, G., \& Corbalán, J. (2010). Creativity, intelectual abilities and response styles, implications for academic performance in the secondary school. Anales de Psicología, 26(2), 212-219.

López-Calichs, E. (2006). El proceso de formación de las competencias creativas. Una necesidad para hacer más eficiente el aprendizaje de los estudiantes universitarios. Revista Iberoamericana de Educación, 40(3), 1-10.

López Martínez, O. y Martín Brufau, R. (2010). Estilos de pensamiento y creatividad. Anales de Psicología, 26(2), 254258.

López Martínez, O. y Navarro, J. (2008) Estudio comparativo entre medidas de creatividad, TTCT vs. CREA. Anales de psicología, 24(1), 138-142.

Mannell, R. (2007). Leisure, Health and Well-Being. World Leisure Journal, 49(3), 114-128.

Martínez Zaragoza, F. (2003). Características Psicométricas del CREA (inteligencia creativa). Un estudio con población española y argentina. RIDEP, 16(2), 71-83.

McKay, AS., Karwowski, M., \& Kaufman, JC. (2017). Measuring the muses: validating the Kaufman domains of creativity scale (K-DOCS). Psychology of Aesthetics, Creativity, and the Arts, 11(2), 216-231.

Moreno, J., Chiecher, A. y Paoloni, P. (2016). ¿Estudiantes en cambio? Resultados del segundo relevamiento del Laboratorio MIG en la Facultad de Ingeniería. Universidad Nacional de Río Cuarto. Documento de trabajo Laboratorio de Monitoreo de Inserción de Graduados. Facultad de Ingeniería. Universidad Nacional de Rio Cuarto.

Newman, D., Tay, L., \& Diener, E. (2014). Leisure and subjective well-being: A model of psychological mechanisms as mediating factors. Journal of Happiness Studies, 15(3), 555578.

Nicolau, A. (2017). Logros creativos, actividades extraacadémicas y de ocio en la universidad. En Elisondo, R. y Melgar, M. Creatividad y Universidad. Estudios más allá de los muros. (pp. 59-93). Málaga: Eumed.

Panaia, M. (2007). Perspectiva futura para los ingenieros. Perfiles, 2(3), $1-2$.

Panaia, M. (2015) Universidades en cambio: ¿generalistas o profesionalizantes? Buenos Aires: Miño y Dávila.

Paoloni, P., Chiecher, A. y Sanchez, L. (2007) Los alumnos de la Facultad de Ingeniería de la UNRC. Características, perfiles, trayectorias. Documento de trabajo Laboratorio de Monitoreo de Inserción de Graduados. Facultad de Ingeniería. Universidad Nacional de Rio Cuarto.

Pascucci, M. (2015). Los jóvenes universitarios y el ocio. European Scientific Journal, 11(10), 116-127.

Silvia, P., Beaty, R., Nusbaum, E., Eddington, K., \& Levin Kwapil, H. (2014). Everyday creativity in daily life: An experiencesampling study of "little c" creativity. Psychology of Aesthetics, Creativity, and the Arts, 8(2), 183-188.
Spuzic, S., Narayanan, R., Abhary, K., Adriansen, H., Pignata, S., Uzunovic, F., \& Guang, X. (2016) The synergy of creativity and critical thinking in engineering design:The role of interdisciplinary augmentation and the fine arts. Technology in Society, $45,1-7$.

Stebbins, R. (2011). Leisure studies: the road ahead. World Leisure Journal, 53(1), 3-10.

Stebbins, R. (2012). Compromiso temporal discrecional: efectos sobre la elección y el estilo de ocio. ARBOR Ciencia, Pensamiento y Cultura, 188(754), 293-300.

Xiaoxia, Ai (1999). Creativity and academic achievement: an investigation of gender differences. Creativity Research Journal, 12(4), 329-337.

Wolfradt, U., \& Pretz, J. (2001). Individual differences in Creativity: Personality, Story Writing and Hobbies. European Journal of Personality, 15, 271-310. 